\title{
Detection of a Bovine Group C Rotavirus from Adult Cows with Diarrhea and Reduced Milk Production
}

\author{
Takahiro MAWATARI ${ }^{1}$, Atsushi TANEICHI ${ }^{1)}$, Takashi KAWAGOE ${ }^{1)}$, Mie HOSOKAWA ${ }^{1)}$, Katsuhiro TOGASHI ${ }^{1)}$ and \\ Hiroshi TSUNEMITSU ${ }^{2) *}$ \\ ${ }^{1)}$ Yamagata Central Livestock Hygiene Center, Urushiyama, Yamagata 990-2161 and ${ }^{2)}$ Shichinohe Research Unit, National Institute of \\ Animal Health, Shichinohe, Aomori 039-2586, Japan
}

(Received 14 November 2003/Accepted 19 February 2004)

ABSTRACT. Only two strains (Shintoku and porcine-like WD534tc) of group C rotavirus (GCR) from cattle have been reported to date. A GCR designated the Yamagata strain was the only pathogen detected in an outbreak of adult cow diarrhea accompanied by a decrease in milk production. The nucleotide sequences of the VP6 and VP7 genes from strain Yamagata were determined. Comparative sequence analysis showed that the sequence identities between strains Yamagata and Shintoku were markedly high in both VP6 gene (98.1\%) a nd VP7 gene (93.5\%), and that these strains belonged to the same clusters which were distinguished from GCRs from different host sp ecies in phylogenetic trees of these genes. These results suggested strongly that cattle species is one of the natural hosts of GCR infection, and that GCRs are a cause of adult cow diarrhea.

KEY WORDS: cattle, diarrhea, group $\mathrm{C}$ rotavirus.

J. Vet. Med. Sci. 66(7): 887-890, 2004

Rotaviruses are members of the family Reoviridae and a major cause of viral diarrheas in humans and animals [11]. At present, rotaviruses are assigned to 6 groups (A to $\mathrm{F}$ ) based on antigenic and genomic analyses [3, 16]. Each group possesses its own group antigen and shows particular double-stranded RNA electrophoretic migration pattern, which is characteristic to each group. The majority of group A rotaviruses (GARs) are differentiated based on the origin of host species (such as human rotavirus, bovine rotavirus and equine rotavirus) although natural GAR cross-species infection and disease have been identified [11, 13, 18]. A similar distinction may exist for other rotavirus groups, although limited numbers of non-GAR strains have been characterized due to the difficulty in propagating most nonGARs in cell culture [3, 16].

Group C rotaviruses (GCRs) were first recognized as a causative agent of diarrhea in piglets [1], after which several reports identified an association between porcine GCRs and diarrhea in nursing and post-weaning pigs [3, 16]. Human GCRs have been associated with sporadic cases and outbreaks of diarrhea in several countries [3, 12]. In cattle, GCR antibodies in sera have been detected in several countries $[2,19]$. We previously isolated the GCR Shintoku strain from adult cow diarrhea in Japan [23], and found that this strain could be distinguished from porcine and human GCRs by antigenic and genetic analyses [8-10, 20, 21]. However, no other reports on the detection of GCR from cattle have appeared, except for the WD534tc porcine-like strain, which is genetically and antigenically more closely related to a porcine GCR than to strain Shintoku [4]. In the present study, we detected a GCR (designated the Yamagata

\footnotetext{
* Correspondence to: Tsunemitsu, H., Shichinohe Research Unit, National Institute of Animal Health, Shichinohe, Aomori 0392586, Japan.
}

strain) from feces of adult cows in an outbreak of diarrhea with a decrease in milk production in Japan. We also conducted a comparative sequence analysis of the VP6 and VP7 genes from strain Yamagata to clarify the genetic relationship of strain Yamagata among GCRs.

An outbreak of diarrhea in adult cows occurred at a dairy farm with 122 lactating Holstein cows in Yamagata Prefecture, Japan. On April 25, 2002, a number of lactating cows suddenly developed diarrhea, with all lactating cows on the farm doing so within several days. The diarrheal feces were liquid and brownish, but not bloody. All affected cows recovered from the diarrhea within 3 to 5 days, and no diarrhea was seen from May 5. A significant decrease in daily milk production on the farm was observed from the day following the first finding of diarrhea and continued for one week. The lowest production (about $10 \%$ reduction) was observed on the third day of the first finding of diarrhea. The affected cows showed no respiratory symptoms. Nonlactating young cattle at the farm showed no clinical signs, including diarrhea. Further, no outbreaks of diarrhea were observed in neighborhood dairy farms at that time.

Samples of diarrheal feces were collected from 3 affected cows. These samples were examined for viruses by electron microscopy (EM), RNA-polyacrylamide gel electrophoresis (RNA-PAGE), reverse transcription-polymerase chain reaction (RT-PCR), and sequencing of PCR products. They were also tested for GAR and adenovirus using antigen detection kits (Dipstic-Rota and Dipstic-Adeno; Eiken, Tokyo, Japan), for Salmonella species and Escherichia coli using standard techniques, for pathogenic factors (LT, ST, VT, ENV, and eaeA) of Escherichia coli by PCR [7], and for Coccidium species and Cryptosporidium species by a sucrose floatation method. For EM, 20\% fecal suspensions were stained with $2 \%$ ammonium molybdate. 
Viral RNA was extracted from the feces using the SepaGene RV-R (Sanko Junyaku, Tokyo, Japan) according to the manufacturer's instructions and used for RNA-PAGE and RT-PCR. For RNA-PAGE, electrophoresis was conducted in $10 \%$ polyacrylamide gels, and gels were stained with a silver stain kit (Bio-Rad, Tokyo, Japan). For GCR detection by RT-PCR, the full-length VP7 gene was amplified with a primer pair corresponding to the 5' and 3' ends of strain Shintoku VP7 gene [20]. RT-PCR for GAR, group $B$ rotavirus (GBR), bovine viral diarrhea virus (BVDV) and bovine coronavirus (BCV) detections were performed as described previously [5, 22, 24, 25]. For amplification of the full-length VP6 gene of GCR, a primer pair corresponding to the common 5' and 3' ends of VP6 gene was used [9]. The PCR products for GCR VP6 and VP7 genes from one sample were sequenced directly by cycle sequencing with an auto sequencer (ABI PRISM 310; Applied Biosystems, Tokyo, Japan). The 5' and 3' end sequences comprising 1824 nucleotides (nt) were derived from the primer sequences. Sequence data were analyzed by the Clustral W method using the Lasergene software (DNASTAR, Madison, WI, US). An unrooted phylogenetic tree was constructed using the neighbor-joining method [17], and drawn with the TreeView program [14].

Rotavirus-like particles were observed in the feces from 1 of 3 affected cows by EM, but no other viruses were observed. All samples were negative for GAR and adenovirus by the antigen detection kits. The EM-positive sample displayed the typical GCR RNA electropherotype (4-3-2-2 pattern) that was closely similar to that of GCR strain Shintoku (Fig. 1). This and one other sample were positive for GCR by RT-PCR. In contrast, all samples were negative for GAR, GBR, BCV, and BVDV in the RT-PCR. These results confirmed that the rotavirus in the feces was GCR, and we designated this virus as the Yamagata strain. All samples were negative for Salmonella species, Coccidium species, Cryptosporidium species, and pathogenic factors of Escherichia coli.

The VP7 gene of GCR strain Yamagata was $1063 \mathrm{nt}$ in length and contained a single ORF encoding 332 amino acids (aa) (DDBJ database accession number: AB108681). The sequence of the VP7 gene from strain Yamagata was then compared with those of the published cognate genes from 1 bovine, 3 porcine, and 6 human GCR strains. A high level of sequence identity was observed between the Yamagata and bovine Shintoku VP7s $(93.5 \%$ in nt and $96.1 \%$ in aa) (Table 1). By contrast, the VP7 sequence identities between strains Yamagata and porcine or human GCR were low (73.9-76.1\% in nt and 72.6-75.0\% in aa).

The strain Yamagata VP6 gene was 1352 nt long and contained one ORF encoding 395 aa (DDBJ database accession number: AB 108680). Table 2 shows the VP6 sequence identities of strain Yamagata with 7 published GCR strains. As with the VP7 gene, the VP6 gene of strain Yamagata was most closely related to that of bovine GCR strain Shintoku, with identities of $98.1 \%$ in nt and $99.5 \%$ in aa (Table 2).

Phylogenetic tree analysis indicated that the VP6 genes of

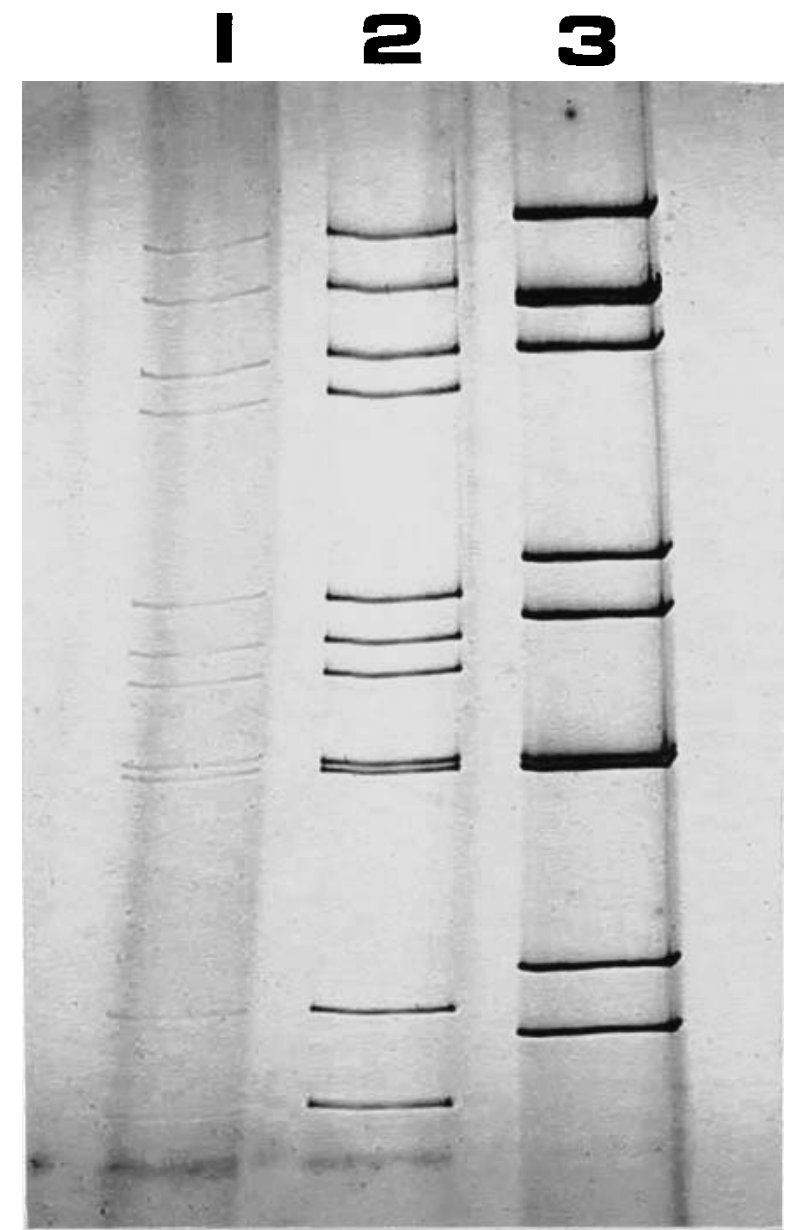

Fig. 1. Comparison of the electrophoretic migration patterns of rotavirus dsRNA. Lane 1: GCR strain Yamagata; Lane 2: GCR strain Shintoku; Lane 3: GAR strain OSU. Migration is from top to bottom

strains Yamagata and Shintoku belonged to the same cluster, as did their VP7 genes. In addition, the VP6 genes were separated into 3 clusters, with each cluster coinciding with the species of origin (Fig. 2).

More than a decade ago, we reported one outbreak of adult cow diarrhea associated with GCR strain Shintoku infection [23]. In that case also, a decline in milk production was observed in diarrheal cows, but detailed information about this could not be obtained. Since that finding, no further reports on outbreaks of GCR-associated diarrhea in cattle have appeared. Thus, little clinical or epidemiological information on GCR infection in cattle is available. In the present case, GCR designated strain Yamagata was the only enteropathogen detected from fecal samples of affected cows, suggesting that GCR strain Yamagata might be associated with the present disease. The disease findings resembled those of the previous GCR infection, and were characterized by acute onset of liquid diarrhea in adult cows 
Table 1. VP7 nucleotide and amino acid sequence identities of strain Yamagata with other published GCR strains

\begin{tabular}{llcc}
\hline & & \multicolumn{2}{c}{ Identity with Yamagata VP7 (\%) } \\
\cline { 3 - 4 } Strain $^{\text {a) }}$ & Species of origin & Nucleotide & Amino acid \\
\hline Shintoku & Bovine & 93.5 & 96.1 \\
Cowden & Porcine & 76.1 & 74.7 \\
WH & Porcine & 75.5 & 75.0 \\
HF & Porcine & 73.9 & 72.6 \\
$88-220$ & Human & 74.2 & 73.5 \\
OK450 & Human & 74.2 & 73.5 \\
Bristol & Human & 74.7 & 73.5 \\
208 & Human & 74.7 & 72.9 \\
Moduganari & Human & 75.1 & 73.8 \\
Jajeri & Human & 74.8 & 73.8 \\
\hline
\end{tabular}

a) The accession numbers of the nucleotide sequences of VP7 genes used are Shintoku, U31750; Cowden, M61101; WH, U31749; HF, U31748; 88-220, M61100; OK450, D87544; Bristol, X77257; 208, AB008671; Moduganari, AF323979; and Jajeri, AF323982.

Table 2. VP6 nucleotide and amino acid sequence identities of strain Yamagata with other published GCR strains

\begin{tabular}{llcc}
\hline & & \multicolumn{2}{c}{ Identity with Yamagata VP6 (\%) } \\
\cline { 3 - 4 } Strain $^{\text {a) }}$ & Species of origin & Nucleotide & Amino acid \\
\hline Shintoku & Bovine & 98.1 & 99.5 \\
WD534tc & Porcine? & 81.6 & 87.8 \\
Cowden & Porcine & 82.9 & 91.6 \\
Bristol & Human & 81.9 & 88.9 \\
208 & Human & 81.7 & 88.6 \\
Moduganari & Human & 81.9 & 88.4 \\
Jajeri & Human & 81.7 & 88.6 \\
\hline
\end{tabular}

a) The accession numbers of the nucleotide sequences of VP6 genes used are Shintoku, M88768; WD534tc, AF162434; Cowden, M94157; Bristol, X59843; 208, AB008672; Moduganari, AF325805; and Jajeri, AF325805.

b) This strain was detected from cattle feces but was thought to be originally from pigs because it was genetically and antigenically closely related to the porcine GCR strain Cowden, and pathogenic to pigs but not cattle [4].

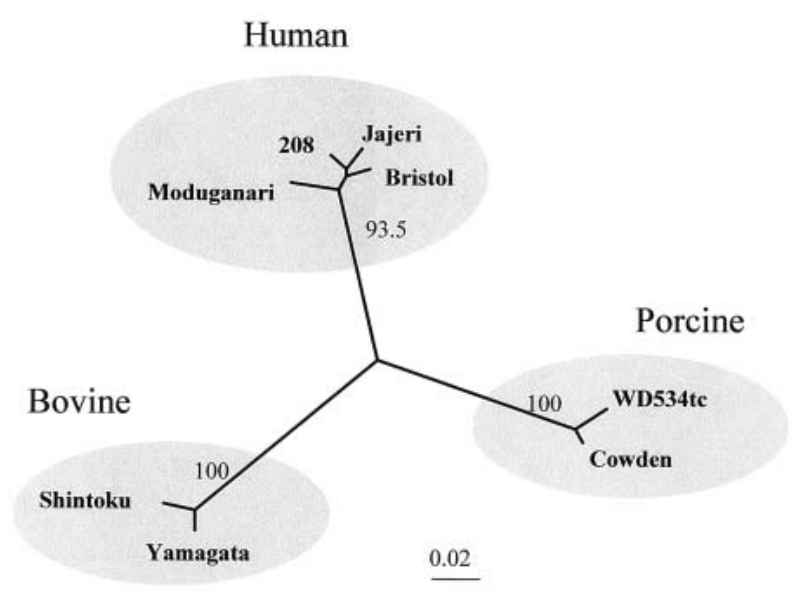

Fig. 2. Unrooted phylogenetic tree showing the relationship of GCR VP6 genes. The boostrap values in 1000 pseudoreplicates for major lineages are shown as percentages. The accession numbers of the nucleotide sequences used refer to the footnote of Table 2. accompanied by a decline in milk production. Diarrhea spread rapidly within the farm with very high morbidity, but no mortality was observed. In contrast, non-lactating young cattle at the same farm did not show diarrhea. These characteristics are closely similar to those of BCV infection [15] and also bovine GBR infection $[6,22]$, which in turn indicates the possibility that GCR infection may have been overlooked clinically in adult cow diarrhea. Thus, laboratory diagnosis including GCR-testing is needed to define the etiological role of GCR infection in adult cow diarrhea. The reason why GCR-associated diarrhea was observed only in adult Holstein cows in both of the present and previous cases is unknown, but lactation might be associated with GCR infection or appearance of diarrhea.

Although GCR antibodies in cattle sera have been detected in several countries with a prevalence of $24-56 \%$ $[2,19]$, to date only GCR strains Shintoku and WD534tc have been detected in cattle. Strain Shintoku was genetically distinguished from other GCRs from different host species based on sequence analysis [8-10, 20]. In addition, strain Shintoku was antigenically distinguished from other 
GCRs by virus neutralization tests [21]. Furthermore, strain Shintoku was pathogenic to calves in a challenge test [19]. In contrast, strain WD534tc was antigenically and genetically closely related to the porcine GCR strain Cowden. Further, strain WD5434tc was pathogenic to pigs but not to calves [4]. Thus, it was considered that the natural host species of strain WD534tc was pigs rather than cattle [4].

In the present study, a close relationship between strains Yamagata and Shintoku was observed genetically by RNAPAGE and comparative sequence analysis of VP6 and VP7 genes. These results strongly suggested that strains Yamagata and Shintoku have evolved from a very recent common ancestor. Furthermore, strains Yamagata and Shintoku belong to the same VP7 genotype which may constitute a single VP7 serotype on the basis of the VP7 sequence identities [20].

In conclusion, cattle species is one of the natural hosts of GCR infection, and strains Yamagata and Shintoku can be called bovine GCRs. GCRs might be a causative agent of adult cow diarrhea. The reason for the discrepancy between the moderate prevalence of GCR antibodies and the small number of reports on GCR detection in cattle may relate to the fact that rotaviruses are generally not investigated in adult cows rather than to any putative asymptomatic status of most GCR infection in cattle. Studies on the prevalence of GCR antibodies among different age groups in cattle are needed to clarify whether the risk of GCR infection in cattle is age-related.

ACKNOWLEDGEMENT. The authors thank Mr. T. Shouji and Mr. T. Onodera for technical assistance.

\section{REFERENCES}

1. Bohl, E. H., Saif, L. J., Theil, K. W., Agnes, A. G. and Cross, R. F. 1982. J. Clin. Microbiol. 15: 312-319.

2. Bridger, J. C. 1987. Ciba Found. Symp. 128: 5-23.

3. Bridger, J. C. 1994. pp. 369-407. In: Viral Infection of the Gastrointestinal Tract, 2nd ed. (Kapikian, A. Z. ed.), Marcel Dekker, New York.

4. Chang, K. O., Nielsen, P. R., Ward, L. A. and Saif, L. J. 1999. J. Virol. 73: 9284-9293.

5. Gouvea, V., Glass, R. I., Woods, P., Taniguchi, K., Clark, K.
F., Forrester, B. and Fang, Z. 1990. J. Clin. Microbiol. 28: 276-282.

6. Hayashi, M., Nagai, M., Hayakawa, Y., Takeuchi, K. and Tsunemitsu, H. 2001. Vet. Rec. 149: 331-332.

7. Itou, F., Yamaoka, K., Ogino, T. and Kanbe, M. 1995. Jpn. J. Clin. Pathol. 43: 772-775.

8. Jiang, B., Gentsch, J. R., Tsunemitsu, H., Saif, L. J. and Glass, R. I. 1999. Virus Genes 19: 85-88.

9. Jiang, B., Tsunemitsu, H., Gentsch, J. R., Glass, R. I., Green, K. Y., Qian, Y. and Saif, L. J. 1992. Virology 190: 542-547.

10. Jiang, B., Tsunemitsu, H., Gentsch, J. R., Saif, L. J. and Glass, R. I. 1993. Nucleic Acids Res. 21: 2250.

11. Kapikian, A. Z., Hoshino, Y. and Chanock, R. M. 2001. pp. 1787-1833. In: Fields Virology, 4th ed. (Knipe, D. M. and Howley, P. M. eds.), Lippincott Williams \& Wilkins, Philadelphia.

12. Kuzuya, M., Fujii, R., Hamano, M., Yamada, M., Shinozaki, K., Sasagawa, A., Hasegawa, S., Kawamoto, H., Matsumoto, K., Kawamoto, A., Itagaki, A., Funatsumaru, S. and Urasawa, S. 1998. J. Clin. Microbiol. 36: 6-10.

13. Nakagomi, O. and Nakagomi, T. 2002. Res. Vet. Sci. 73: 207214.

14. Page, R.D. 1996. Comput. Appl. Biosci. 12: 357-358.

15. Saif, L. J. 1990. Cornell Vet. 80: 303-311.

16. Saif, L. J. 1990. pp. 73-95. In: Viral Diarrhea of Man and Animals (Saif, L. J. and Theil, K. W. eds.), CRC press, Boca Raton.

17. Saitou, N. and Nei, M. 1987. Mol. Biol. Evol. 4: 406-425.

18. Taniguchi, K., Urasawa, T. and Urasawa, S. 1994. Virology 200: $390-400$

19. Tsunemitsu, H., Jiang, B. and Saif, L. J. 1992. J. Clin. Microbiol. 30: 2129-2134.

20. Tsunemitsu, H., Jiang, B. and Saif, L. J. 1996. Arch. Virol. 141: 705-713.

21. Tsunemitsu, H., Jiang, B., Yamashita, Y., Oseto, M., Ushijima, H. and Saif, L. J. 1992. J. Clin. Microbiol. 30: 3009-3012.

22. Tsunemitsu, H., Morita, D., Takaku, H., Nishimori, T., Imai, K. and Saif, L. J. 1999. Arch. Virol. 144: 805-815.

23. Tsunemitsu, H., Saif, L. J., Jiang, B. M., Shimizu, M., Hiro, M., Yamaguchi, H., Ishiyama, T. and Hirai, T. 1991. J. Clin. Microbiol. 29: 2609-2613.

24. Tsunemitsu, H., Smith, D. R. and Saif, L. J. 1999. Arch. Virol. 144: $167-175$.

25. Vilcek, S., Herring, A. J., Herring, J. A., Nettleton, P. F., Lowings, J. P. and Paton, D. J. 1994. Arch. Virol. 136: 309-323. 